\title{
The evolution of Nova V382 Velorum 1999
}

\author{
M. Della Valle ${ }^{1}$, L. Pasquini ${ }^{2}$, D. Daou ${ }^{3}$, , and R. E. Williams ${ }^{4}$ \\ 1 Osservatorio Astrofisico di Arcetri, Largo E. Fermi 5, Firenze, Italy \\ e-mail: massimo@arcetri.astro.it \\ 2 European Southern Observatory; KarlSwarschildstrasse 2, Garching bei München, München, Germany \\ e-mail: lpasquin@eso.org \\ 3 California Institute of Technology MS: 220-6 1200 East California Blvd., Pasadena, CA 91125, USA \\ e-mail: daou@ipac.caltech.edu \\ ${ }^{4}$ Space Telescope Science Institute, 3700 San Martin Drive, Baltimore, MD, USA \\ e-mail: wms@stsci.edu
}

Received 25 February 2002 / Accepted 22 April 2002

\begin{abstract}
We report results of spectroscopic observations of V382 Vel (Nova Vel 1999) carried out at La Silla between 5 and 498 days after maximum light (23 May 1999, $V(\max ) \sim 2.3 \pm 0.1$ ). The analysis of the photometric and spectroscopic evolution shows this object to be a fast nova belonging to the Fe II broad spectroscopic class. A distance of $1.7 \mathrm{kpc}( \pm 20 \%)$ is derived from the maximum magnitude vs. rate of decline relationship after correcting for the small reddening toward the nova, $E(B-V) \lesssim 0.10$. From the measured $\mathrm{H} \alpha$ flux and the associated rate of expansion we derive an approximate mass for the ejected shell, $M_{\text {env }} \lesssim 10^{-5} M_{\odot}$. We have also observed during the early decline a broad, short-lived ( $\lesssim 2$ weeks) feature at 6705-6715 ̊ for which several identifications are possible, one of which is the lithium doublet at $6708 \AA$ and which could place an empirical limit on the lithium production that might occur during the outburst of a fast nova. The high luminosity at maximum, $M_{\mathrm{v}}=-8.9$, and the relatively small height above the galactic plane $(z \lesssim 160 \mathrm{pc})$ suggest that V382 Vel originated from a massive white dwarf, likely in the mass range $1.1-1.2 M_{\odot}$.
\end{abstract}

Key words. stars: novae, cataclysmic variables

\section{Introduction}

Nova Velorum 1999 (=V382) was independently discovered by P. Williams (1999) and A. Gilmore (1999), on May 22, as a star brighter than $V \sim 3$, located at $\mathrm{RA}=10^{\mathrm{h}} 44^{\mathrm{m}} 48^{\mathrm{s}} .39$, Decl. $=-52025^{\prime} 30$ ! 7 (equinox 2000.0, Garrad 1999). Early spectroscopy obtained by Lee et al. (1999) confirmed the object to be a classical nova undergoing outburst. V382 Vel achieved optical maximum roughly 1 day later. This brightness makes V382 Vel the brightest galactic nova since V1500 Cyg 1975 and one of the brightest novae of the century (see Table 1), thus offering us a unique opportunity to study its photometric and spectroscopic evolution in great detail. Its spectroscopic evolution was followed at La Silla Observatory as an ESO target of opportunity with the $1.5 \mathrm{~m}$ telescope equipped with FEROS (Fiber-fed Extended Range Optical Spectrograph), a high-resolution echelle spectrograph characterized by high efficiency and large wavelength range, covering the entire optical spectral range (3800-9000 $\mathrm{A}$ ) in a single format at a resolution of $R=48000$.

Send offprint requests to: M. Della Valle, e-mail: massimo@arcetri.astro.it
Table 1. Brightest Novae since 1900.

\begin{tabular}{cccc}
\hline \hline$\#$ & Nova & Year & $V(\max )$ \\
\hline 1. & v603 Aql & 1918 & -1.1 \\
2. & GK Per & 1901 & 0.2 \\
3. & CP Pup & 1942 & 0.5 \\
4. & RR Pic & 1925 & 1.0 \\
5. & DQ Her & 1934 & 1.3 \\
6. & v476 Cyg & 1920 & 1.6 \\
7. & v1500 Cyg & 1975 & 1.8 \\
8. & CP Lac & 1936 & 2.1 \\
9. & V382 Vel & 1999 & 2.3 \\
10. & v533 Her & 1963 & 2.5 \\
11. & Q Cyg & 1876 & 3.0 \\
& v446 Her & 1960 & 3.0 \\
\hline
\end{tabular}

\section{Lightcurve}

The lightcurve of the nova and its color evolution are shown in Fig. 1 using the photometric measurements of Gilmore \& Kilmartin (1999) with the $0.6 \mathrm{~m}$ telescope at Mount John University Observatory. An extrapolation of the photometric measurements near maximum light indicates that V382 Vel reached $V=2.3 \pm 0.1$ on 23 May 1999 . 


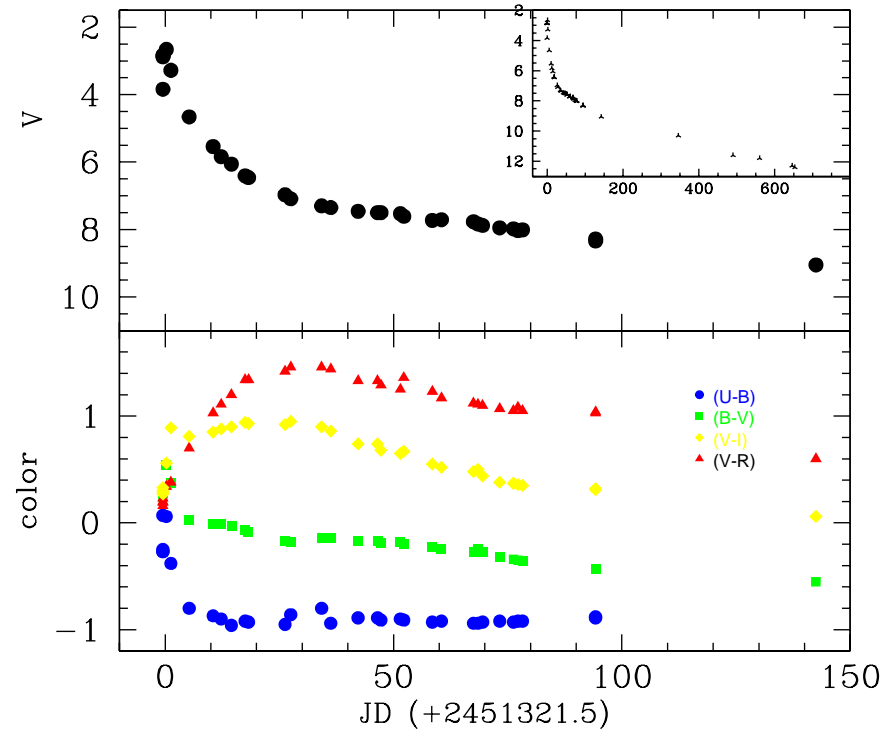

Fig. 1. The lightcurve of v382 Vel (top) and its color evolution (bottom).

The nova decreased in brightness by two magnitudes from maximum in $t_{2}=4.5$ days $\left(t_{3}=9 \mathrm{~d}\right)$. Applying these data to the maximum magnitude vs. rate of decline relationship derived by Della Valle \& Livio (1995) for novae in M 31 and the LMC indicate that V382 Vel was intrinsically luminous, achieving an absolute magnitude at maximum of $M_{V}=-8.9 \pm 0.17$. The observed $(B-V)$ obtained by averaging 4 measurements at maximum light yields $\langle(B-V)\rangle=0.28 \pm 0.03$. This is very similar to the value $(B-V)=0.23 \pm 0.06$ obtained by van den Bergh $\&$ Younger (1987) for a sample of unreddened galactic novae, suggesting that V382 Vel suffered little galactic absorption $\left(A_{V} \lesssim 0.1-0.15 \mathrm{mag}\right)$. Similar conclusions were drawn by Shore et al. (1999a) after noting the lack of the 217.5-nm interstellar feature in STIS spectra of the nova. The evolution of the colors is very typical, becoming bluer with time as the ejecta become optically thin and the photosphere recedes. The smoothness of the lightcurve during decline suggests little dust formation (if any) in the ejecta.

The mean magnitude of the nova at quiescence has been determined by Platais et al. (1999) by examing photographic plates from the Yale/San Juan Southern Proper Motion program. They derived $V=16.56 \pm 0.13$ and an average $(B-V)=$ 0.14 , which is consistent with the unreddened colors of novae at minimum (cf. Bruch 1984). The outburst amplitude of $\Delta M \sim 14.3$ magnitudes is larger than the average outburst for galactic novae ( $\sim 12 \mathrm{mag}$ ), but is not unusual for fast novae (for example, CP Pup 1942 and V1500 Cyg 1975 exhibited amplitudes of $\gtrsim 16$ mag and $\gtrsim 19 \mathrm{mag}$, respectively). This amplitude and the derived absolute magnitude at maximum lead to $M_{V} \sim 5.3$ at minimum, which falls in the faint tail of the distribution for classical novae (see Della Valle \& Duerbeck 1993, their Fig. 3).

Platais et al. (1999) also point out the discovery on a photographic plate obtained on 28 April 1970 of a flaring of 0.5 magnitudes. Such flaring is not uncommon in the years before a major outburst in classical novae (e.g. Robinson 1975).

\section{Spectroscopic observations}

\subsection{The early decline}

Spectroscopic observations of the nova began at ESO five days past maximum as part of a target of opportunity campaign (Della Valle et al. 1999). We have for the first time exploited the potential of the ESO $1.5 \mathrm{~m}$ telescope equipped with FEROS (see Kaufer et al. 1999) on a bright galactic nova, and have collected a series of 13 spectra in the optical range 3800-9000 $\AA$ with a resolution of 48000 . Five representative spectra are shown in Fig. 2. The first spectrum, obtained on 28 May, exhibits a bluish continuum dominated by Balmer emission, Fe II and other low ionization heavy element lines, in addition to two absorption systems, the "Principal" and "Diffuse Enhanced" absorption normally observed in early decline (Payne-Gaposchkin 1957). The expansion velocities for these systems measured from the minima of the Balmer line P-Cyg profiles are close to 2300 and $3700 \mathrm{~km} \mathrm{~s}^{-1}$ respectively, and the HWZI measured for the $\mathrm{H} \alpha$ emission feature is $3600 \mathrm{~km} \mathrm{~s}^{-1}$. $\mathrm{H} \alpha$ and $\mathrm{H} \beta$ show rather asymmetric "saddleshaped" profiles (see Fig. 3), with the blue component clearly more prominent than the red. This difference disappears with time, and between 3 and 6 June the blue component diminishes, as the profiles evolve to a flat-topped shape. At the same time the steady decrease in the expansion velocity of the emitting gas (Fig. 4), initially decreasing as a power law in time $\left[\sim \Delta t^{n}\right.$ with $n$ varying from $-0.32(\mathrm{H} \alpha)$ to $-0.15(\mathrm{H} \beta)$ and $-0.10(\mathrm{H} \delta), \Delta t=$ time since maximum light], changes character as it reaches $\Delta t \sim 10$ days slowly decreasing during the following $\sim 400$ days by $\sim 10-15 \%$ only. During this interval (the initial 15 days past maximum) the nova decreases in brightness by more than 3 magnitudes from maximum. The identifications and relative intensities of all emission lines observed in the spectra between 28 May 1999 and 2 Oct. 2000 are given in Table 3 . The line identifications are provided in Col. 1, whereas the intensity of each line has been determined, independently by two of us, by fitting its profile (or deconvolved profile in the case of blends) with multiple Gaussian fits in MIDAS (Banse et al. 1988) (using the Alice package) and IRAF environments. Intensities are given relative to $\mathrm{H} \beta$ (Cols. 2-14). The FEROS spectra were not calibrated in absolute flux on the nights that observations were obtained, so a relative FEROS response curve was obtained from the spectra of spectrophotometric standards observed during commissioning (Kaufer et al. 1999). The high stability of the instrument and the large diameter fibre aperture (2.5 arcsec) result in a stable instrument response function that can be used even though not obtained on the same nights as the observations. Absolute fluxes were determined by matching the observed energy distributions with the corresponding $B$ and $V$ photometric measurements (cf. Table 2). The most striking change in the spectrum occurs one month after maximum light when the early low ionization permitted transitions give way to emerging higher ionization and forbidden lines. This is accompanied by a change to flat-topped line profiles, probably associated with the termination of the post- outburst wind phase and the complete ejection of the envelope. 


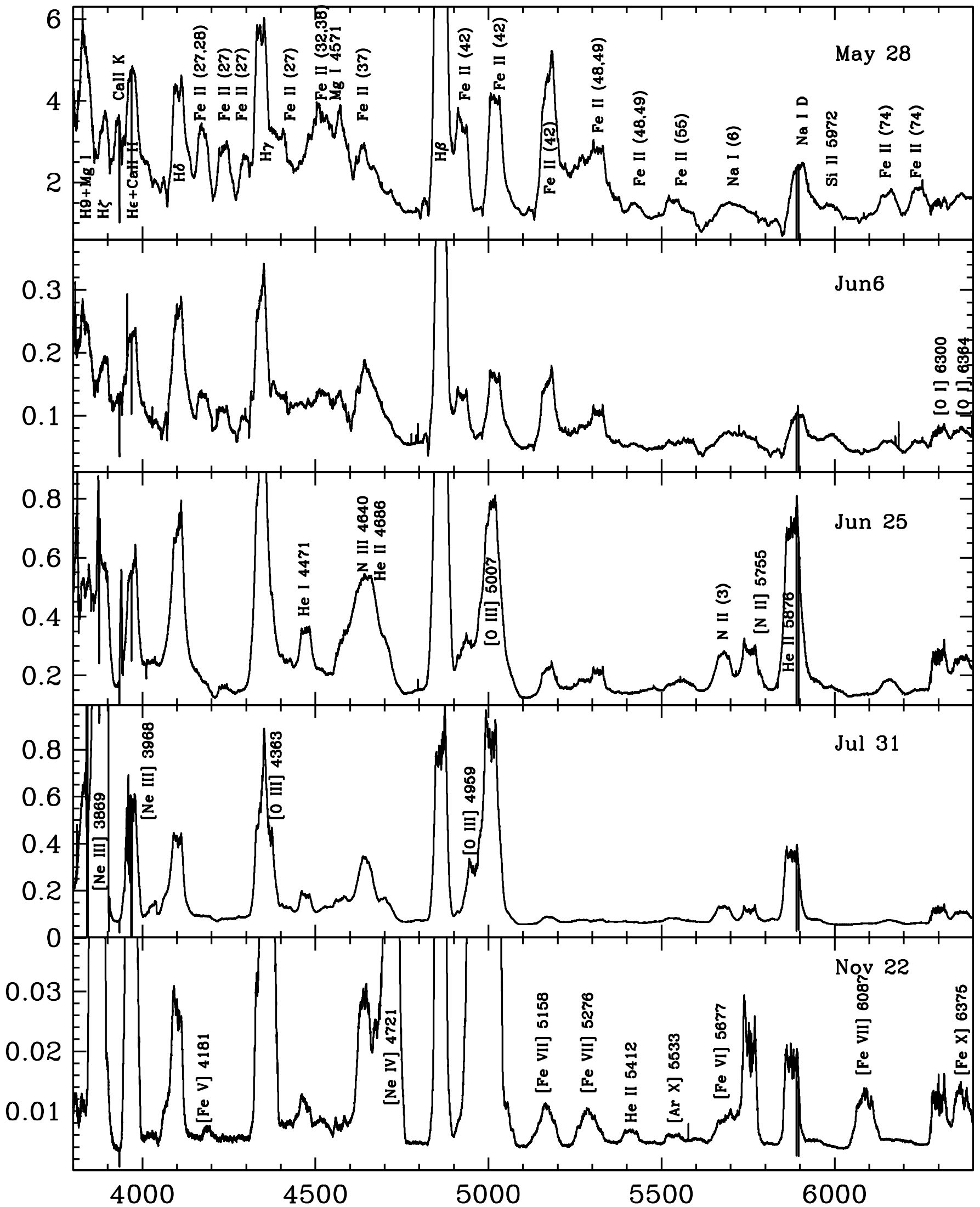

Fig. 2. The spectroscopic evolution of v382 Vel 1999. Spectra obtained (from top) on May 28, Jun. 6, Jun. 25, Jul. 31 and Nov. 22 , 1999. 


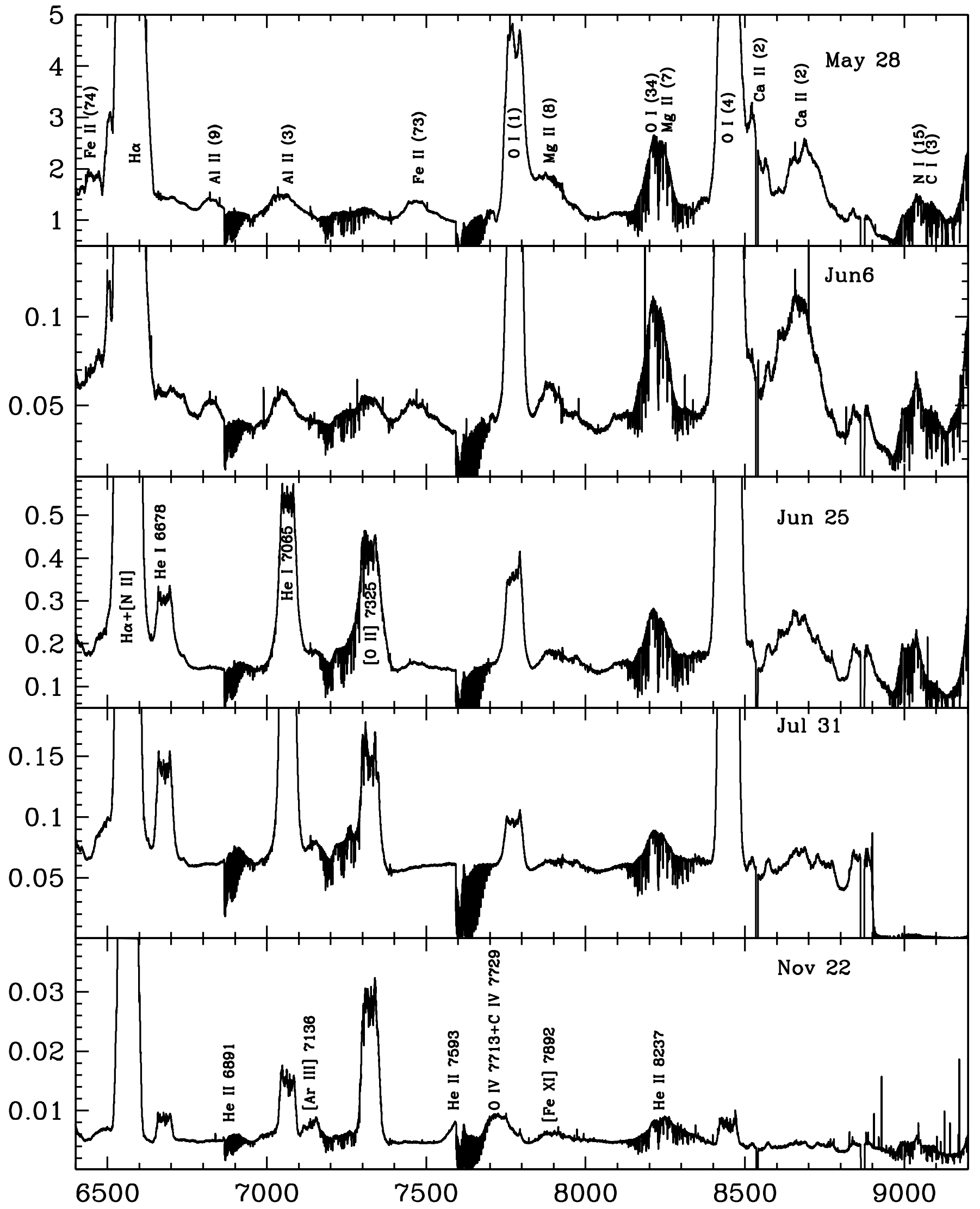

Fig. 2. continued. 

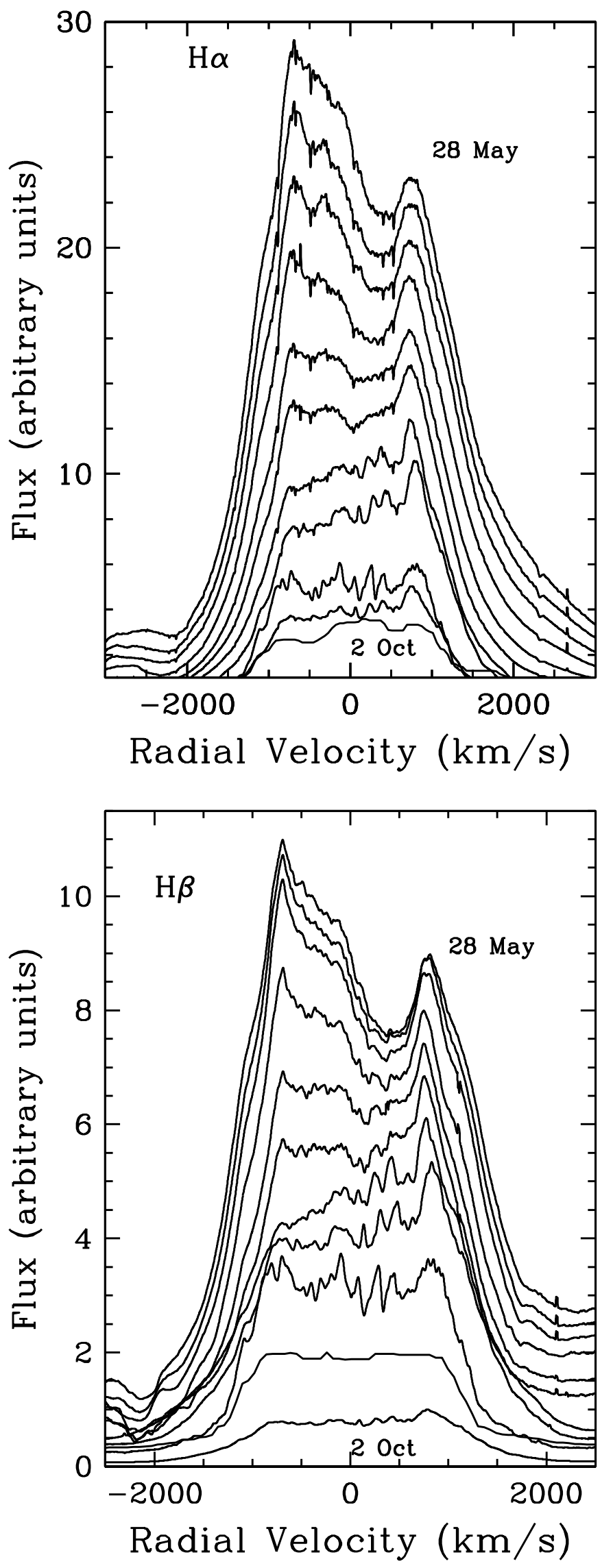

Fig. 3. The evolution of the $\mathrm{H} \alpha$ and $\mathrm{H} \beta$ profiles with the time. From top, 28, 29, 30 May, 3, 6, 25 Jun., 19, 31 Jul., 22 Nov. 1999, and 2 Oct. 2000 .

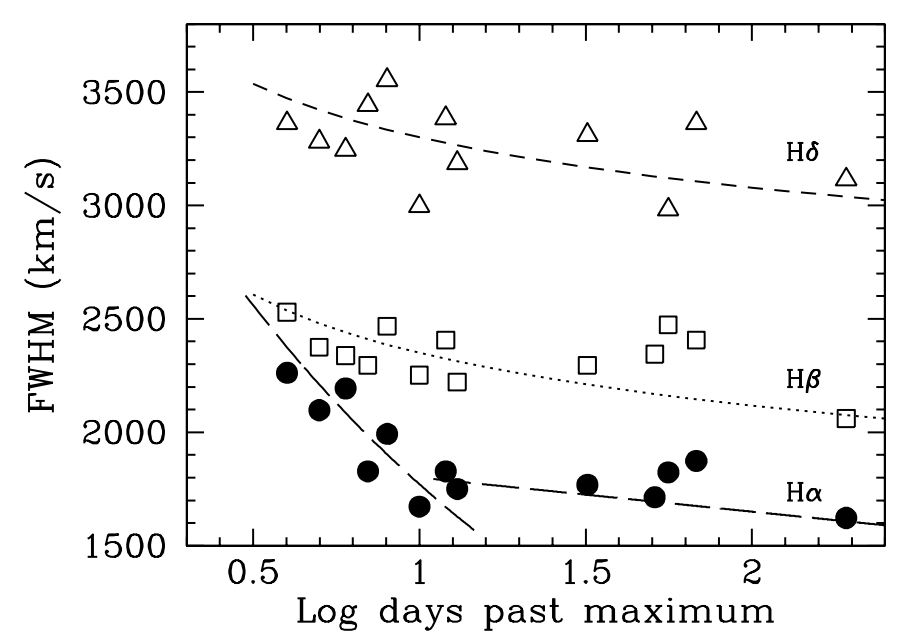

Fig. 4. The evolution of the $F W H M$ of $\mathrm{H} \alpha, \mathrm{H} \beta, \mathrm{H} \delta$ with the time.

Table 2. $\mathrm{H} \beta$ fluxes: erg $\mathrm{cm}^{-2} \mathrm{~s}^{-1}$.

\begin{tabular}{cccc}
\hline \hline Date & $\Delta t$ & $F_{\mathrm{H} \beta}$ & $\%$ error \\
\hline 28 May 99 & 5 & $2.54 \mathrm{e}-11$ & 0.39 \\
29 May 99 & 6 & $2.20 \mathrm{e}-11$ & 0.30 \\
30 May 99 & 7 & $1.54 \mathrm{e}-11$ & 0.25 \\
1 Jun. 99 & 9 & $1.13 \mathrm{e}-11$ & 0.21 \\
2 Jun. 99 & 10 & $8.96 \mathrm{e}-12$ & 0.17 \\
3 Jun. 99 & 11 & $1.02 \mathrm{e}-11$ & 0.28 \\
5 Jun. 99 & 13 & $5.34 \mathrm{e}-12$ & 0.42 \\
6 Jun. 99 & 14 & $3.50 \mathrm{e}-12$ & 0.29 \\
25 Jun. 99 & 33 & $2.89 \mathrm{e}-12$ & 0.06 \\
14 Jul. 99 & 53 & $1.82 \mathrm{e}-12$ & 0.07 \\
19 Jul. 99 & 58 & $1.63 \mathrm{e}-12$ & 0.37 \\
31 Jul. 99 & 69 & $1.07 \mathrm{e}-12$ & 0.22 \\
22 Nov. 99 & 183 & $3.89 \mathrm{e}-13$ & 0.45 \\
2 Oct. 00 & 498 & $5.62 \mathrm{e}-14$ & 0.30 \\
\hline
\end{tabular}

\subsection{The nebular stage}

The nova entered the nebular spectral stage by the end of June, roughly 40 days after maximum light. At that time the spectra were characterized by the increasing strengths of [O III] $\lambda \lambda 4959,5007 \AA$ and He I lines, and the gradual disappearance of the Fe II lines and the absorption systems. The spectra obtained on Nov. 22, 1999 and Oct. 2, 2000 show [O III] brighter than $\mathrm{H} \alpha+[\mathrm{N} \mathrm{II}]$ and exhibiting a flat-top, castellated structure with a HWZI of $\sim 2000 \mathrm{~km} \mathrm{~s}^{-1}$, with the He I lines fading and soon disappearing. During this stage we note also the emergence of strong [Ne III] $\lambda \lambda 3869,3968 \AA$, and [Fe VII] $\lambda \lambda 6087 \AA$, which are typical of the nebular stages of novae belonging to the $\mathrm{He} / \mathrm{N}$ and Fe II "broad" spectroscopic class (see Williams 1992).

\section{Interstellar extinction}

As previously mentioned the nova showed little evidence for extinction near maximum light. An independent estimate of the reddening at subsequent times can be made from a comparison of predicted and observed emission lines ratios within the same ion which are not sensitive to radiative transfer effects (Robbins 1968a,b). Unblended H and He recombination 


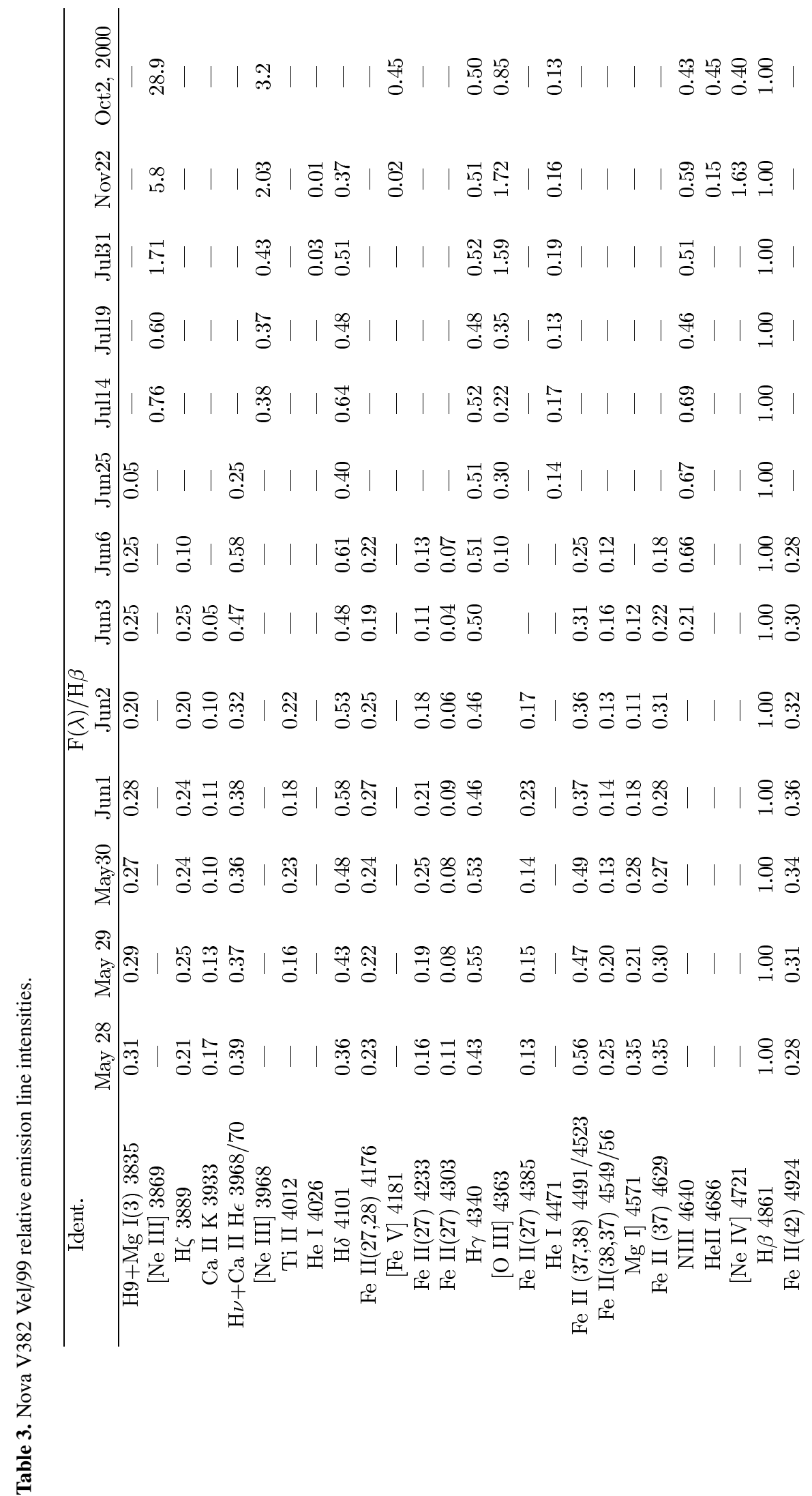




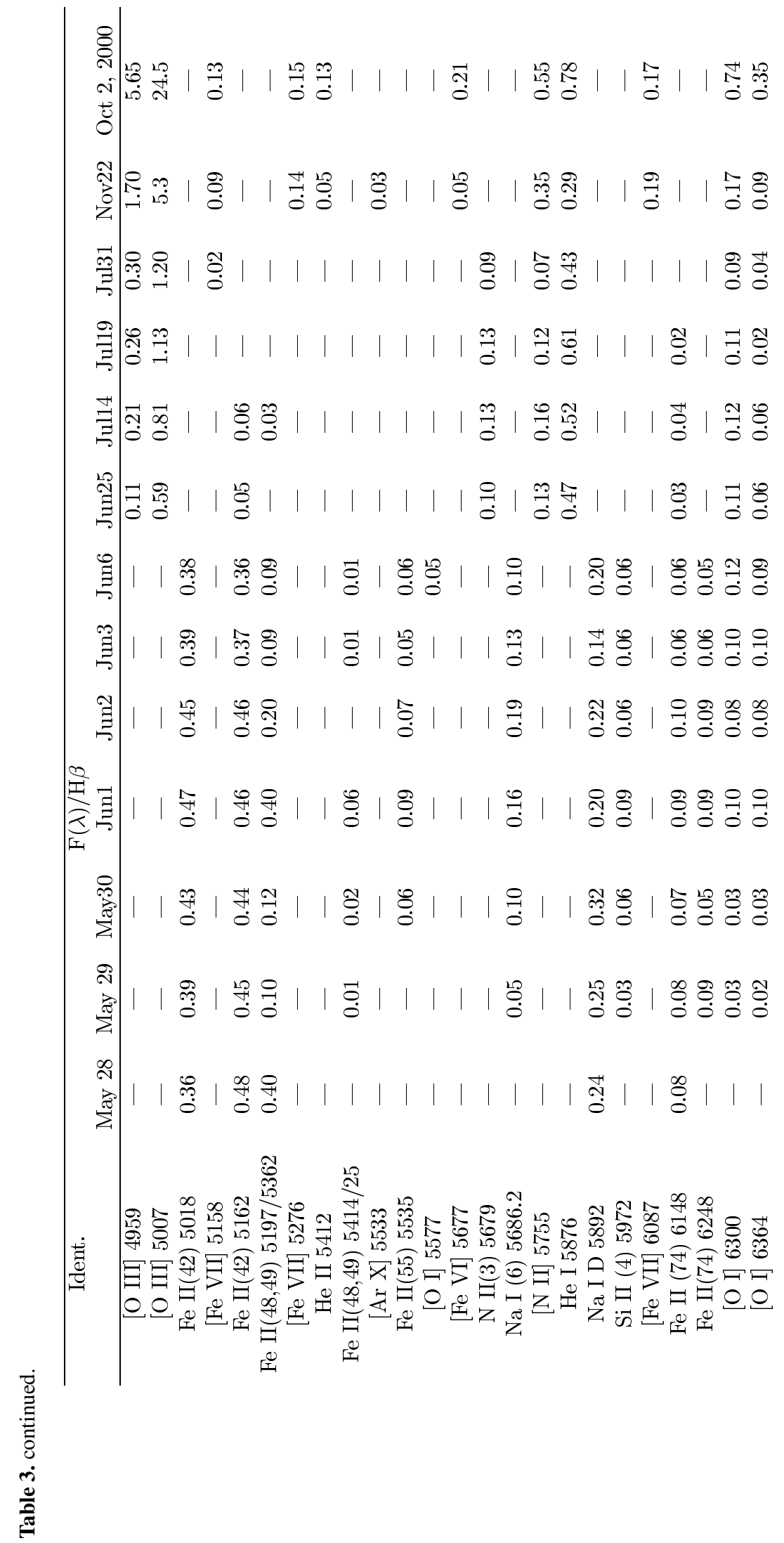




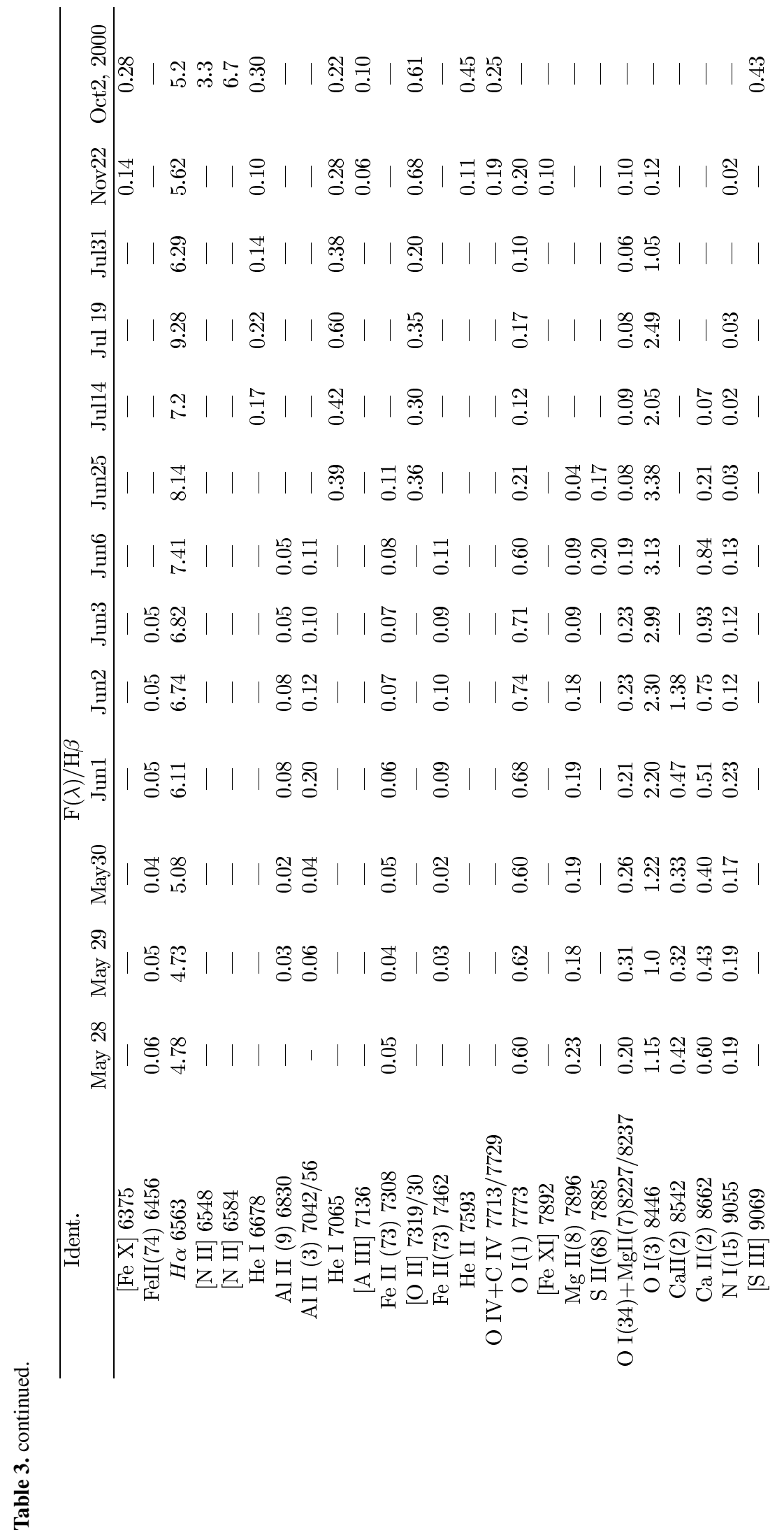


lines may be used for this purpose if they are optically thin and not affected by collisions. Because $\mathrm{H} \alpha$ is seriously blended with the [NII] lines the HeI lines offer the best possibility for this purpose, and data presented in Table 3 provide ratios at 6 epochs with a median value of $\lambda 5876 / \lambda 4471=3.06$, which can be compared with the recombination value of 2.9. Using the ratio of total to selective absorption $R=3.2$ (Seaton 1979), one derives $E_{B-V}=0.05$ (neglecting collisional effects). A separate value of reddening can be derived from the $\lambda 6678 / \lambda 4471$ ratio. The observed value (median from 5 epochs) of 0.77 is close to the theoretical value of 0.78 .

All nova spectra show clear insterstellar $\mathrm{NaI} \mathrm{D}$ and $\mathrm{Ca}$ II $\mathrm{H}$ and $\mathrm{K}$ absorption. Na D1 and D2 are saturated, and the minimum intensity of the lines is zero intensity, however the $\mathrm{Ca}$ II $\mathrm{H}$ line does show some residual intensity. For both doublets only one absorption component is seen at our resolution. The equivalent widths of the lines as measured from our FEROS spectra are 360 and $395 \mathrm{~mA}$ for the D1 and D2 lines, and 203 and $382 \mathrm{~m} \AA$ for $\mathrm{H}$ and $\mathrm{K}$ of CaII. The IS component has a velocity of $4.4 \mathrm{~km} \mathrm{~s}^{-1}$ and the width of the lines (given as the $F W H M$ of the Gaussian fitting of the different lines), is 0.23 and $0.25 \AA$ for Ca II H and K (17.4 and $19.1 \mathrm{~km} \mathrm{~s}^{-1}$ ), and 0.322 and 0.345 angstrom for the D1 and D2 components (16.4 and $\left.17.6 \mathrm{~km} \mathrm{~s}^{-1}\right)$. By applying crude estimates for the reddening vs. $\mathrm{Na} \mathrm{D}$ equivalent widths, e.g. $E(B-V)=0.13 \times E W(\mathrm{D}$ lines $)$ (Benetti, private communication) we find $E(B-V) \sim 0.09$. Although this scaling relation is approximate, a comparison with the $\mathrm{Na} E W$ observed in detailed IS studies with resolved single components (e.g. the SN 1987a field Molaro et al. 1993) indicates a reddening slightly higher than that estimated from emission line ratios, viz., $A_{V} \lesssim 0.3 \mathrm{mag}$. In general, however, all methods confirm a rather low reddening and therefore a moderate distance to the nova.

\section{The $[\mathrm{O} I]$ and $[\mathrm{O} \mathrm{III]} \mathrm{lines}$}

Measurements of the intensities of emission lines in nova spectra have shown an anomalous ratio of the [O I] $\lambda \lambda 6300,6363 \AA$ nebular lines (see Williams 1994). The relative intensities of these lines is usually not 3:1 (an exception was FH Ser 1970, see Rosino et al. 1986), as expected from their atomic transition probabilities. Nova Vel 1999 is no exception as the data shown in Table 3 averaged over 11 epochs lead to a $\lambda 6300 / 6363$ ratio of $1.8 \pm 0.3$. Williams (1994) has discussed this situation in novae, and has suggested that optically thick [O I] lines must be formed in very dense $\left(n(\mathrm{H}) \gtrsim 10^{14} \mathrm{~cm}^{-3}\right)$, small blobs of neutral material embedded within the ionized ejected shells.

During early decline when the density of the ejecta is in the high-density limit of the [O I] lines the intensity ratio $F_{\lambda 6300} / F_{\lambda 5577}$ can be used to determine the temperature (e.g. Osterbrock 1989) from the relation:

$F_{\lambda 6300} / F_{\lambda 5577}=0.023 \times\left(1-\mathrm{e}^{-\tau}\right) / \tau \times \exp \left(25800 / T_{\mathrm{e}}\right)$.

The [O I] line at $\lambda 5577$ is clearly detected in the spectra of on 5 and 6 June with $F_{\lambda 6300} / F_{\lambda 5577}=2.19$. This is within the range of measured intensity ratios reported by Williams (1994), although close to the lower limit of the sample, and implies $T_{\mathrm{e}}(\mathrm{K})=4800$.
The evolution of the nebular and auroral [O III] emission lines, $F_{\lambda(4959+5007)} / F_{\lambda 4363},(\sim 1$, on 31 July $1999 ; \sim 3.1$, on 22 November 1999; and 236.7 on 2 October 2000), suggests (cf. Filippenko \& Halpern 1984) for the typical range of temperatures $3.4 \lesssim \log T_{\mathrm{e}} \lesssim 3.7$ (see Williams 1994, his Table 3), values of the density initially (31 July) close to the high density limit $n_{\mathrm{e}} \sim 10^{9} \mathrm{~cm}^{-3}$, and eventually decreasing to $n_{\mathrm{e}} \sim 10^{4} \mathrm{~cm}^{-3}$.

\section{The mass of the ejected envelope}

The most reliable distances to classical novae are parallaxes derived from angular size measurements of the expanding ejecta. Currently this method can be applied to about thirty galactic novae (out of more than 200 objects discovered to date), therefore most nova distance measurements involve the use of the maximum magnitude vs. rate of recline relationship. First noted by Zwicky (1936), it is characterized by an intrinsic scatter of 0.16 mag $(1 \sigma)$, (Della Valle \& Livio 1995) corresponding to a distance uncertainty of $\sim 20 \%(3 \sigma)$. The photometric data presented in the introduction yield a reddening-corrected distance modulus $(m-M)=11.04$, which corresponds to a distance of $1660 \pm 110(1 \sigma) \mathrm{pc}$. The emitted luminosity of $\mathrm{H}_{\alpha}$ follows from the distance, from which an estimate of the mass of the ionized hydrogen content in the ejecta can be made. The $\mathrm{H} \alpha$ emission from the envelope is $E \alpha=g_{\alpha} \times n_{\mathrm{e}}^{2} \times \epsilon \times{\text { Volume } \mathrm{erg}^{-1}}^{-1}$ where $g_{\alpha} \gtrsim 10^{-24} \mathrm{erg} \mathrm{cm}^{3} \mathrm{~s}^{-1}$ is the emission coefficient per $\mathrm{cm}^{3}$, for $T \lesssim 5000$ (and $n_{\mathrm{e}}=1$ ) and $\epsilon$ the volume filling factor. Assuming the volume of the expanding shell to be $V \sim 4 \pi \times R^{2} \times \delta$, where $R \sim v_{\exp } \times \Delta t(\Delta t=498$ days from the maximum light and $v_{\exp }=1600 \mathrm{~km} \mathrm{~s}^{-1}$ ) and $\delta=f R$ (with $f$ smaller than 1) is the thickness of the shell. From the data of Tables 2 and 3 (2 Oct. 2000), we obtain:

$n_{\mathrm{e}}^{2}=\frac{7 \times 10^{6}}{\delta \epsilon}$

If we assume the thickness of the envelope increases from thermal motions inside the gas (Mustel \& Boyarchuk 1970), then for $T \sim 5000 \mathrm{~K}, f \sim v_{\text {ther }} / v_{\text {exp }} \lesssim 0.2$ and then $n_{\mathrm{e}} \lesssim 10^{4} \mathrm{~cm}^{-3}$, which yields $6.5 \times 10^{-6} M_{\odot}$. This is an order of magnitude smaller than ejecta mass determinations for slow novae, but not unusual for fast novae. Indeed being $\left\langle\Delta m_{\text {eject }}\right\rangle \propto R_{\mathrm{WD}}^{4} / M_{\mathrm{WD}}$ and $M_{B}(\max ) \propto \log M_{\mathrm{WD}}$ (Livio 1992), the mass of the shell should correlate with the rate of decline (in view of the existence of the Maximum Magnitude vs. Rate of Decline relationship), as we actually observe, at $\sim 95 \%$ confidence level (see Fig. 5)

$\log \Delta m_{\text {eject }}=0.274( \pm 0.197) \times \log t_{2}+0.645( \pm 0.283)$.

However we note that the previous calculation refers to the $\mathrm{H}$ ionized mass, therefore our estimate should be regarded as a lower limit for the mass of the ejected envelope.

Observations of the [O I] line intensities formed when the density in the ejecta exceeds critical density, after correction for reddening and optical depth effects, yield the oxygen mass of the neutral component into the ejected shell when the distance and the electron temperature are known (e.g. Williams 1994). The previous data give $M\left(\mathrm{O}^{0}\right) \sim 9.3 \times 10^{-8} M_{\odot}$ which is only $\sim 1 \%$ of the total mass of the shell. On the other hand, 


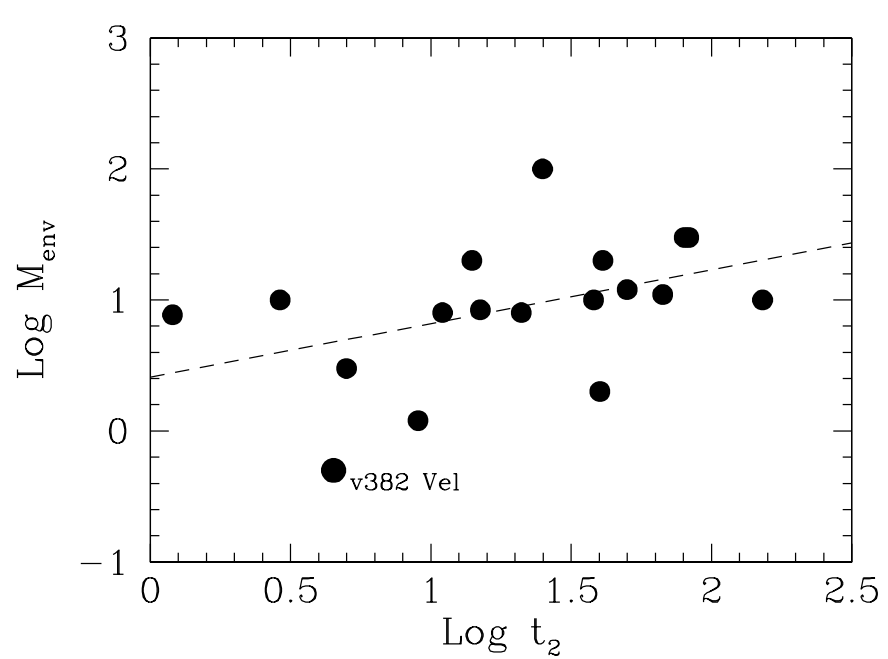

Fig. 5. The correlation between the rate of decline and the mass of the ejected envelope. Data from Gehrz (1988), Snijders et al. (1987), Hjellming (1990) (V1500 Cyg), Gehrz (1988), Stickland et al. (1981) (v1668 Cyg); Hjellming (1990), Hartwick \& Hutchings (1978) (HR Del); Ferland et al. (1984), Martin (1989) (DQ Her); Woodward et al. (1992) (v838 Her); Pottasch (1959), Ferland (1979) (CP Lac); Gehrz (1988), Hassal et al. (1990) (GQ Mus); Williams \& Gallagher (1979) (RR Pic); Hartwick \& Hutchings (1978), Hjellming (1990), Della Valle et al. (1997) (FH Ser); Gehrz (1988) (LW Ser); Raikova (1990) (LV Vul); Gehrz (1988) (NQ Vul); Gehrz (1988), Saizar et al. (1991) (PW Vul); Taylor et al. (1988), Saizar et al. (1992) (QU Vul); Gehrz et al. (1993) (QV Vul), de Freitas Pacheco (1977), Ferland (1979) (IV Cep), de Freitas Pacheco et al. (1989) (v842 Cen); Snijders et al. (1987) (v1370 Aql).

this should be regarded as a lower limit for the total amount of oxygen present in the ejecta (typically $\lesssim 15 \%$, see Gehrz et al. 1998 ), because this procedure does not include the contribution of other $\mathrm{O}$ ions.

\section{The short-lived feature at $6705-6715 \AA$}

A close inspection of the early spectra reveals the presence at $\sim 6700 / 6715 \AA$ of a weak and broad emission line (see Fig. 6) which increases in strength during the early decline and reaches maximum intensity about 10 days past maximumm, disappearing within a few days (undetectable on the Jun. 25 spectrum.) The measured FWZI corresponds to an expansion velocity of $\sim 1100 \mathrm{~km} \mathrm{~s}^{-1}$, about one third of the velocity measured for the Balmer emission lines. The identification of this feature is not straightforward and its narrowness suggests that it could be a component of the broader feature that is immediately blueward. The measured wavelength and rapid evolution with time and its presence during early decline require that it originates from a low ionization ion (e.g. Si, N, Ni, Al). There is no unambiguous identification for this feature, since no abundant ion has a strong transition at this wavelength, however one possibility is the $6708 \AA$ doublet of Li I.

Novae have been predicted to be sources of galactic lithium (e.g. Romano et al. 1999 and reference therein, although see Travaglio et al. 2001 for a different view), however attempts to detect lithium (see Friedjung 1979) have been unsuccessful. According to Starrfield et al. (1978) and Boffin et al. (1993)

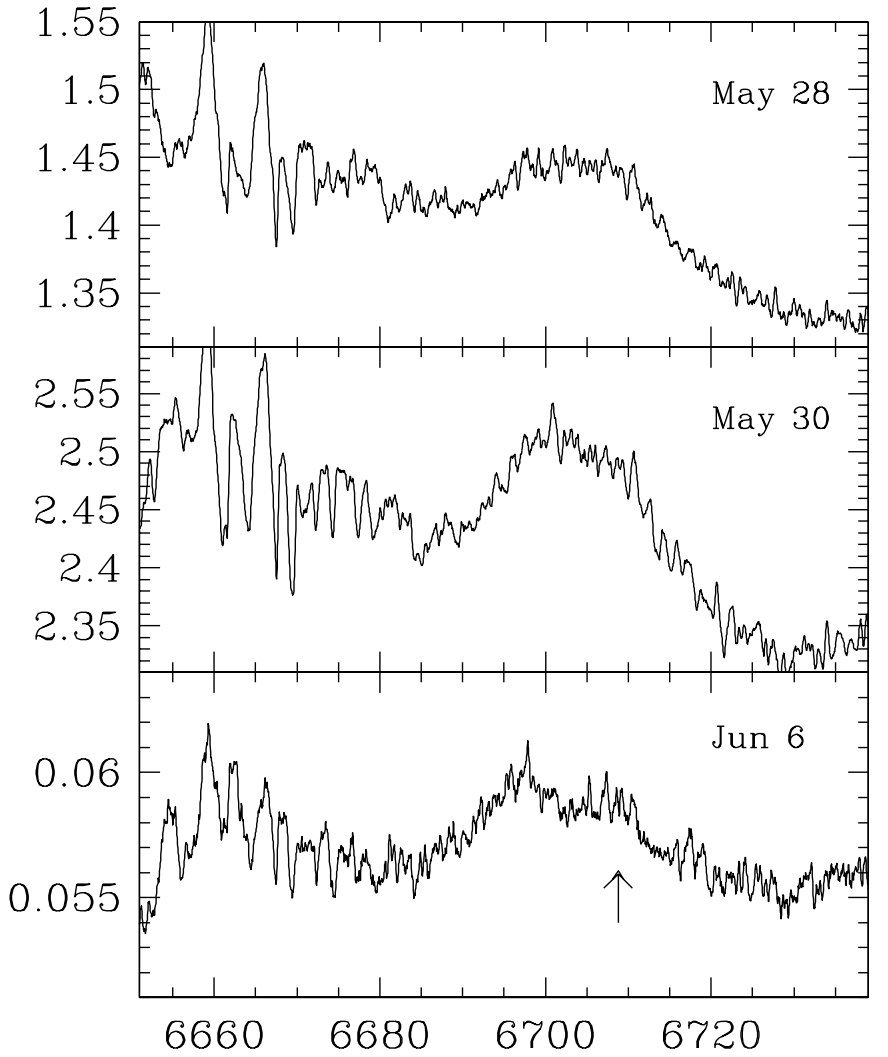

Fig. 6. The evolution of the broad feature at $6705-6715 \AA$.

(see also Norgaard \& Arnould 1975) Li should be produced by the radioactive decay of ${ }^{7} \mathrm{Be}$, whose half-life time is 53 days, and therefore it is not expected to be detectable during the earliest stages of a nova. This constraint may be relaxed if one assumes that $\mathrm{Li}$ begins to form prior to the peak of TNRs and the observed variation of the intensity of the line during the early nova evolution is dominated by ionization effects in the ejecta.

The observed intensity of the unidentified feature with respect to the emission lines of hydrogen is $\gtrsim 10^{-6}$. Assuming an abundance of $\mathrm{H}$ in the ejected shell of $\sim 10^{-5 /-6} M_{\odot}$ (see above) the unidentified feature represents at most an injection of $\mathrm{Li}$ into the interstellar medium of $\sim 10^{-11 /-12} M_{\odot}$, if it is formed by recombination. If $10^{4}$ is the average number of outbursts experienced by a nova during its lifetime (Bath \& Shaviv 1978), then about $\sim 10^{-7 /-8} M_{\odot}$ could be assumed as an upper limit to the possible production of Li during the lifetime of a fast nova. This value is appoximately the theoretical prediction of lithium produced by a nova during its lifetime (D'Antona \& Matteucci 1991; Romano et al. 1999).

\section{Conclusions}

According to the quantitative criteria defined by Della Valle \& Livio (1998) there are two classes of novae drawn from two populations of progenitors:

a) Slow Novae achieve an absolute magnitude of $M_{B} \lesssim$ -7.5 at maximum, their lightcurves exhibit a slow decline (often characterized by secondary maxima) with $t_{3} \gtrsim 20$ days (or $t_{2} \gtrsim 12$ days), and they normally belong to the Fe IIn 
( $\mathrm{n}=$ narrow) spectroscopic class defined by Williams (1992). They occur at distances of up to $\gtrsim 1 \mathrm{kpc}$ above the galactic plane and are probably related to the Pop II stellar population of the bulge/thick disk, and are therefore associated with less massive white dwarfs, $M_{\mathrm{WD}} \lesssim 0.9-1 M_{\odot}$;

b) Fast Novae have lightcurves characterized by very bright peak luminosities, $M_{B} \lesssim-9$, followed by rapid, smooth declines ( $t_{3} \lesssim 20$ days, or $t_{2} \lesssim 12$ days), and they mostly belong to the $\mathrm{He} / \mathrm{N}$ or $\mathrm{Fe} \mathrm{IIb}$ spectroscopic classes defined by Williams (1992). The progenitors originate from a relatively old Pop I stellar population of the thin disk/spiral arm, and therefore they are located close to the galactic plane, typically $z \lesssim 100-200 \mathrm{pc}$. The associated white dwarfs are rather massive, $M_{\mathrm{WD}} \gtrsim 0.9-1 M_{\odot}$.

Nova Vel 1999 was the brightest nova to occur in the Milky Way in the last 25 years, and the rate of decline implies that this nova achieved an absolute magnitude of $M_{B} \sim-9$, suggesting that it probably originated from a massive white dwarf, with $M_{\mathrm{WD}} \sim 1.15 M_{\odot}$, and likely originating from an O-NeMg WD (see Shore et al. 1999a,b). We also note: $a$ ) its location relatively close to the galactic plane, $z \lesssim 160 \mathrm{pc} ; b$ ) the fast and smooth decline $\left(t_{2}=4.5^{\mathrm{d}}, t_{3}=9^{\mathrm{d}}\right)$, without the secondary maxima or sudden variations in brightness (e.g. DQ Her 1934) that are indicative of dust formation in the ejecta; $c$ ) quick evolution to the nebular stage ( $\sim 40$ days, i.e. from $1 / 3$ to $1 / 10$ of the time required by a typical slow nova; $d$ ) the presence of strong Fe II lines, characterized (to start from phase $\sim 10$ days, i.e. at the time when most envelope had been already ejected) by almost "flat-topped" profiles and high expansion velocities ( $\gtrsim 3500 \mathrm{~km} \mathrm{~s}^{-1}$ at half of FWZI), indicating that this nova was forming the emission lines in a discrete shell, and $e$ ) the presence during the nebular stage of forbidden lines of high ionization, such as [Fe VII], [Fe X] and [Fe XI]. All of this toghether points out that this nova belongs to the broad Fe II class (Fe IIb) of Williams (1992) scheme of spectroscopic classification (see also Williams et al. 1994) and it qualify as one of the best examples of a prototypical fast nova.

The analysis of the spectroscopic data has shown the existence of a short-lived ( $\$ 2$ weeks) emission feature in the region $6700-6715 \AA$. There is no unambiguous identification for this feature, and one possibility is the $6708 \AA$ lithium doublet. Novae are believed to be sources of Li for the galactic ISM, and the intensity of this emission line implies $\sim 10^{-11 /-12} M_{\odot}$ as an upper limit to the lithium produced by a fast nova in a single outburst, a value that is near the theoretical predictions currently circulating in literature (see Romano et al. 1999; Jose \& Hernanz 1998 and references therein).

We have also estimated the mass of ionized hydrogen in the envelope from the emitted flux in $\mathrm{H} \alpha$, and under simple geometrical assumptions we obtain $M_{\text {env }} \gtrsim 6.5 \times 10^{-6} M_{\odot}$ which corresponds to a rather small mass envelope, although not atypical for a fast nova. There is also a considerable uncertainty in this value due to the unknown filling factor. If the filling factor of the envelope is close to $10^{-2 /-4}$ rather than $\sim 1$, as HST observations of the Recurrent Nova T Pyx suggest (Shara et al. 1997), then a large decrease in the mass of the nova ejecta will result. Evidence in favor of a moderately small value for the filling factor is indicated by the high optical depth of [O I] lines for
Nova Vel 1999, which suggest that part of the ejecta are condensed blobs of neutral material immersed in the ionized environment of the shell. Our data (collected on 2 October 2000) may suggest a value for the filling factor as small as $\epsilon \approx 0.1$.

A second source of uncertainty is our estimate of the thickness of the shell. In the past, the simple assumption $f \sim$ $v_{\text {ther }} / v_{\exp }$ has led to underestimates of the thickness of the ejected shells for two cases, DQ Her 1934 (Humason 1940) and FH Ser 1970 (Della Valle et al. 1997) by a factor 3-4.

The relatively small distance to V382 Vel (which partially explains the lack of reddening toward the nova, in spite of the small value of its galactic latitude) and the high expansion velocity of the ejecta, make Nova Vel 1999 a suitable target for HST observations, within a short time. A systematic follow-up of the nova remnant, in $\mathrm{H} \alpha$ and [OIII] emission, coupled with simultaneous ground-based high-resolution spectroscopic observations, would offer us the unique opportunity to study the detailed evolution of the ejecta and to provide a reliable estimate of the mass of envelope with greater accuracy, and thus to properly evaluate the contribution of classical novae to the galactic nucleosynthesis.

Acknowledgements. The authors are deeply grateful to the La Silla staff for having promptly activated the service-mode and providing the spectroscopic follow-up of the nova. MDV thanks Space Telescope Science Institute, where part of this work was done, for its hospitality. The authors are also indebted with F, Matteucci, H. Duerbeck, S. Starrfield, P. Kilmartin and A. Gilmore for helpful discussions and with an anonymous referee for his remarks, which have improved the presentation of this paper.

\section{References}

Banse, K., Ponz, D., Ounnas, C., Grosbol, P., Warmels, R. 1988, in Instrumentation for Ground-Based Optical Astronomy, Santa Cruz 1987, ed. L. B. Robinson (Springer-Verlag, New York), 431 Bath, G. T., \& Shaviv, G. 1978, MNRAS, 183, 515

Boffin, H. M., Paulus, G., Arnould, M., \& Mowlavi, N. 1993, A\&A, 279,173

Bruch, A. 1984, A\&AS, 56, 441

D'Antona, F., \& Matteucci, F. 1991, A\&A, 248, 62

Della Valle, M., \& Duerbeck, H. 1993, A\&A, 271, 175

Della Valle, M., \& Livio, M. 1995, ApJ, 452, 704

Della Valle, M., Gilmozzi, R., Bianchini, A., \& Esenoglu, H. 1997, A\&A, 325, 1151

Della Valle, M., \& Livio, M. 1998, ApJ, 506, 818

Della Valle, M., Pasquini, L., \& Williams, R. E. 1999, IAUC 7193

de Freitas Pacheco, J. A. 1977, MNRAS, 181, 421

de Freitas Pacheco, J. A., da Costa, R. D. D., \& Codina S. J. 1989, ApJ, 347, 483

Ferland, G. J. 1979, ApJ, 231, 781

Ferland, G. J., Williams, R. E., Lambert, D. L., et al. 1984, ApJ, 281, 194

Filippenko, A. V., \& Halpern, J. P. 1984, ApJ, 285, 458

Friedjung, M. 1979, A\&A, 77, 357

Garrad, G. J. 1999, IAUC 7177

Gehrz, R. D. 1988, ARA\&A, 26, 377

Gehrz, R. D., Truran, J. W., \& Williams, R. E. 1993, in Protostars and Planets III, p. 75

Gehrz, R. D., Truran, J. W., Williams, R. E., \& Starrfield, S. 1998, PASP, 110, 3 
Gilmore, A. C. 1999, IAUC 7176

Gilmore, A. C., \& Kilmartin, P. M. 1999, IAUC 7238

José, J., \& Hernanz, M. 1998, ApJ, 494, 680

Kaufer, et al. 1999, The Messeneger, 95, 8

Hartwick, F. D. A., \& Hutchings, J. B. 1978, ApJ, 226, 203

Hassall, B. J. M., Snijders, M. A. J., Harris, A. W., et al. 1990, in Physics of Classical Novae, Proceedings of Colloquium No. 122 of the International Astronomical Union, held in Madrid, Spain, June 1989, ed. A. Cassatella, \& R. Viotti (Publisher, SpringerVerlag, Berlin, Germany), 202

Hjellming, R. M. 1990, Lect. Notes Phys., 369, 169

Humason, M. L. 1940, PASP, 52, 389

Lee, et al. 1999, IAUC 7176

Livio, M. 1992, ApJ, 393, 516

Martin, P. G. 1989, in Classical Novae, ed. M. F. Bode, \& A. Evans (Wiley \& Sons, Chichester)

Molaro, P., Vladilo, G., Monai, S., et al. 1993, A\&A, 274, 505

Mustel, E. R., \& Boyarchuck, A. A. 1970, Ap\&SS, 6, 183

Norgaard, H., \& Arnould, M. 1975, A\&A, 40, 331

Osterbrock, D. E. 1989, in Astrophysics of Gaseous Nebulae and Active Galactic Nuclei (University Science Books)

Payne-Gaposchkin, C. 1957, The Galactic Novae (North-Holland Publishing Company, Amsterdam)

Platais, I., Girard, T. M., Kozhurina-Platais, V., et al. 2000, PASP, 112, 224

Pottasch, S. 1959, Ann. Astrophys., 22, 412

Raikova, D. 1990, Lect. Notes Phys., 369, 163

Robbins, R. R. 1968a, ApJ, 151, 497

Robbins, R. R. 1968b, ApJ, 151, 511
Robinson, E. L., AJ, 80, 515

Romano, D., Matteucci, F., Molaro, P., \& Bonifacio, P. 1999, A\&A, 352,117

Rosino, L., Ciatti, F., \& Della Valle, M. 1986, A\&A, 158, 34

Saizar, P., Starrfield, S., Ferland, G. J., et al. 1991, ApJ, 367, 310

Saizar, P., Starrfield, S., Ferland, G. J., et al. 1992, ApJ, 398, 651

Seaton, M. J. 1979, MNRAS, 187, 73

Shara, M. M., Zurek, D. R., Williams, R. E., et al. 1997, AJ, 114, 258

Shore, S., et al. 1999a, IAUC 7192

Shore, S., et al. 1999b, IAUC 7261

Snijders, M. A. J., Batt, T. J., Roche, P. F., et al. 1987, MNRAS, 228, 329

Starrfield, S., Truran, J. W., Sparks, W. M., \& Arnould, M. 1978, ApJ, 222, 600

Stickland, D. J., Penn, C. J., Seaton, M. J., Snijders, M. A. J., \& Storey, P. J. 1981, MNRAS, 197, 107

Taylor, A. R., Hjellming, R. M., Seaquist, E. R., \& Gehrz, R. D. 1988, Nature, 335, 235

Travaglio, C., Randich, S., Galli, D., et al. 2001, ApJ, 559, 909

van den Bergh, S., \& Younger, P. F. 1987, A\&AS, 70, 125

Williams, P. 1999, IAUC 7176

Williams, R. E., \& Gallagher, J. S. 1979, ApJ, 228, 482

Williams, R. E. 1992, AJ, 104, 725

Williams, R. E., Phillips, M. M., \& Hamuy, M. 1994, ApJS, 90, 297

Williams, R. E. 1994, ApJ, 426, 279

Woodward, C. E., Gehrz, R. D., Jones, T. J., \& Lawrence, G. F. 1992, ApJ, 384, L41

Zwicky, F. 1936, PASP, 48, 191 